\title{
Anomalously Large Thermal Expansion at the (0001) Surface of Beryllium without Observable Interlayer Anharmonicity
}

\author{
K. Pohl,,${ }^{1, *}$ J.-H. Cho, ${ }^{2, \dagger}$ K. Terakura, ${ }^{2}$ M. Scheffler, ${ }^{3}$ and E. W. Plummer ${ }^{1}$ \\ ${ }^{1}$ Solid State Division, Oak Ridge National Laboratory, Oak Ridge, Tennessee 37831-6057 \\ and Department of Physics and Astronomy, The University of Tennessee, Knoxville, Tennessee 37996-1200 \\ ${ }^{2}$ JRCAT, National Institute of Advanced Interdisciplinary Research, 1-1-4 Higashi, Tsukuba, Ibaraki 305, Japan \\ ${ }^{3}$ Fritz-Haber-Institut der Max-Planck-Gesellschaft, Faradayweg 4-6, D-14195 Berlin-Dahlem, Germany
}

(Received 24 November 1997)

\begin{abstract}
We have measured a large thermal surface expansion, 6 times larger than the bulk, on $\mathrm{Be}(0001)$ using low-energy electron diffraction. This observation seems to be inconsistent with previous measurements reporting negligible anharmonicity in the surface phonon modes normal to the surface. Density-functional theory calculations for the thermal expansion from the minimum in the free energy within the quasiharmonic approximation agree with the experimental observations and demonstrate that the enhanced thermal expansion is caused largely by a softening of the in-plane vibrations. [S0031-9007(98)05647-6]
\end{abstract}

PACS numbers: 63.20.Ry, 68.35.Bs, 68.35.Ja

Thermal expansion of a crystal is a direct manifestation of the anharmonic nature of the interatomic forces in solids. For purely harmonic interatomic potentials, the mean positions of the atoms do not change even though their vibrational amplitudes increase with temperature. Thus, an expansion upon heating is intimately related to the anharmonicity of normal modes of lattice vibrations, which, in turn, are determined by the nature of binding between the different units in the lattice. When a crystal is cleaved, a surface is formed by breaking the symmetry and reducing the coordination. This process causes larger thermal motion of the surface atoms and presumably enhanced anharmonicity in the interlayer potential. As the temperature is increased, this anharmonicity can lead to large anisotropic vibrations, surface roughening, and premelting [1].

Early free energy calculations with pair potentials for the (100), (110), and (111) surfaces of $\mathrm{Cu}$ showed that anharmonicity and its effect on the equilibrium atomic positions (thermal expansion) and the vibrations around the equilibrium atomic positions (mean-square displacements) should be 2-3 times larger at the surface as compared to the bulk [2]. The first experimental evidence for enhanced thermal expansion of a crystal surface was reported for the $\mathrm{Pb}(110)$ surface [3] and then for $\mathrm{Ni}(100), \operatorname{Ag}(111)$, and $\mathrm{Cu}(110)$ [4]. The coefficients of thermal expansion were found to be 5 to 20 times larger than in the bulk, much higher than suggested by the aforementioned theory. The assumption in all of these studies was that the enhanced thermal expansion is caused by the greatly enhanced anharmonicity of vibrations perpendicular to the surface.

The first quantitative measurement of surface anharmonicity was performed on $\mathrm{Cu}(110)$ by Baddorf and Plummer [5] using high-resolution electron energy loss spectroscopy (HREELS). In their study, the temperature dependence of the surface phonon energies and life- times revealed a vibrational anharmonicity for motion normal to the surface 4-5 times greater than in the bulk. Unfortunately, there are no equivalent measurements of anharmonicity for $\mathrm{Ag}(111), \mathrm{Ni}(100)$, and $\mathrm{Pb}(110)$ to complement the thermal expansion measurements.

Recently, the density-functional theory calculations by Narasimhan and Scheffler [6] and Cho and Scheffler [7] have challenged the intuitive picture of thermal expansion. They argue that thermal expansion at surfaces is driven largely by a rapid softening of the in-plane phonon frequencies with increasing temperature rather than anharmonicity in the out-of-plane modes. This proposal is physically compelling, because the reduction in symmetry at the surface in combination with the fact that the bulk structure confines the in-plane relaxation will undoubtedly lead to a coupling of the interlayer separation and in-plane motion.

The large top layer expansion of $+5.8 \%$ reported for $\mathrm{Be}(0001)$ [8] at room temperature from low-energy electron diffraction (LEED) is in obvious conflict with first-principles calculations, which found an expansion of $+2.7 \%$ [9] and $+2.5 \%$ [10] for $T=0 \mathrm{~K}$, neglecting zeropoint vibrations. The measurement of the temperature dependence of the Rayleigh wave at the $\mathrm{Be}(0001)$ surface by Hannon [11] found very little anharmonicity in the zone boundary surface phonons at both $\bar{K}$ and $\bar{M}$. Here, the Rayleigh wave consists almost entirely of motion of the top layer atoms normal to the surface, i.e., the interlayer potential shows no sign of enhanced anharmonicity. Since thermal expansion is thought to be intimately associated with anharmonicity, a significant thermal expansion causing the disagreement was prematurely ruled out. It should be pointed out that surface x-ray diffraction cannot be employed for this low $Z$ element. Beryllium is a window material in $\mathrm{x}$-ray beam lines.

In this Letter, we report the measurements of the interlayer spacing at the surface of $\mathrm{Be}(0001)$ as a function 
of temperature and compare our data with densityfunctional theory calculations. We find that the experimental top layer separation expands from $+3.1 \%$ at $110 \mathrm{~K}$ to $+4.3 \%$ at $300 \mathrm{~K}$ and $+6.7 \%$ at $700 \mathrm{~K}$, giving a large surface coefficient of thermal expansion $\alpha_{s}=70 \times 10^{-6} \mathrm{~K}^{-1}$, whereas the bulk value is only $\alpha_{b}=12 \times 10^{-6} \mathrm{~K}^{-1}$. The presented theoretical results agree well with our experimental observation. Moreover, our theory shows that a softening of the in-plane vibrations are more important to the thermal surface expansion than the out-of-plane vibrations. Thus, we present evidence that a surface can exhibit large thermal expansion even though the interlayer potential shows very little anharmonicity.

The clean $\mathrm{Be}(0001)$ surface was prepared by sputteranneal cycles at $700 \mathrm{~K}$ and monitored with HREELS and Auger electron spectroscopy. The sharp $(1 \times 1)$ LEED pattern at normal incidence exhibited a sixfold symmetry indicating a natural averaging over the two possible terminations $(A$ or $B$ ) of the threefold symmetric hcp(0001) surface. The experimental LEED $I$ - $V$ data sets were formed by averaging all recorded equivalent beam intensities as a function of incident electron energy. This resulted in three beam profiles (10), (11), and (20) with a total energy range $\Delta E$ of $970 \mathrm{eV}$. Full sets of LEED $I-V$ data were taken at three different sample temperatures: cooled to $110 \mathrm{~K}$, at $300 \mathrm{~K}$, and resistively heated to $700 \mathrm{~K}$. For the latter measurement, the heating current was cycled and the $I-V$ data were only acquired during periods when the current was zero. The large thermal vibrations at $700 \mathrm{~K}$ allowed for measurement of one beam profile with an energy range of $220 \mathrm{eV}$.

The analysis of the LEED $I-V$ spectra was carried out using standard multiple scattering algorithms [12]. Atomic scattering matrices were calculated from a maximum of 14 phase shifts, which we derived from bulk potentials [13], and were renormalized for thermal vibrations using a Debye temperature $\Theta_{D}$ converted into isotropic mean-square displacements $\left\langle u^{2}\right\rangle_{1},\left\langle u^{2}\right\rangle_{2}$, and $\left\langle u^{2}\right\rangle_{b}$ for the first two layers and the bulk, respectively. Intralayer scattering was treated exactly via matrix inversion and layers were stacked by renormalized forward scattering. Electron attenuation was included by the energy-dependent imaginary part of the optical potential $V_{0 i}$ modeled by the equation $V_{0 i}=-5.0 \mathrm{eV}$ $\left\{\left(E-V_{0 r}\right) /\left(150 \mathrm{eV}-V_{0 r}\right)\right\}^{1 / 3}$, where $E$ is the electron energy. The energy dependence of the real part was assumed to be linear: $V_{0 r}=\alpha+\beta(E-150 \mathrm{eV}), \alpha$ and $\beta$ were optimized during the search procedure. We used the method of tensor-LEED [14] for the structural search. The agreement between measured and calculated $I-V$ curves were quantified by the Pendry $R$ factor $R_{P}$ [15].

Figure 1 shows the experimental data for the (10) beam at the three temperatures studied. The low electron energy part of the spectra show shifts in peak positions, which can be caused only by changes in the surface structure. The excellent agreement with the calculated

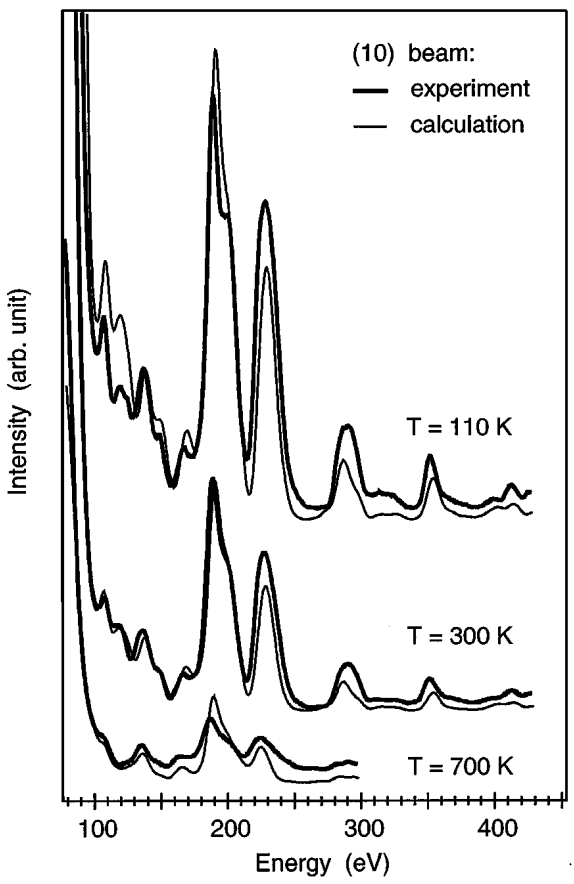

FIG. 1. Comparison of the measured LEED $I-V$ spectra (thick lines) for the (10) beams to the calculated diffraction intensities (thin lines) for the optimized structures at temperatures $T=$ 110,300 , and $700 \mathrm{~K}$. The experiment-calculation $I-V$ curve pairs are arbitrarily shifted vertically but the relative intensity scale is conserved.

$I-V$ curves for the best-fit structures is obvious from the very low values of $R_{P}$ given in Table I. We find a temperature-dependent increase in the top layer expansion from $+3.1 \%( \pm 0.7)$ at $110 \mathrm{~K}$ to $+4.3 \%( \pm 0.7)$ at $300 \mathrm{~K}$ and $+6.7 \%( \pm 1.2)$ at $700 \mathrm{~K}$, which is accompanied by 5 times larger thermal mean-square displacements of the surface atoms compared to the bulk. Figure 2(a) shows the change in the surface relaxations with temperature. It should be noted that the experimental surface expansions are quoted relative to the appropriate bulk expansion,

TABLE I. Geometric parameters extracted from the best fit to the LEED $I-V$ data for the three temperatures investigated. Temperature-dependent bulk lattice constants from $\mathrm{x}$-ray diffraction [23].

\begin{tabular}{cccc}
\hline \hline & $110 \mathrm{~K}$ & $300 \mathrm{~K}$ & $700 \mathrm{~K}$ \\
\hline$a_{0}(T)_{\text {bulk }}(\AA)$ & 2.2826 & 2.2856 & 2.3008 \\
$\Delta d_{12} / d_{0}(\%)$ & $+3.1 \pm 0.7$ & $+4.3 \pm 0.7$ & $+6.7 \pm 1.2$ \\
$\Delta d_{23} / d_{0}(\%)$ & $+1.4 \pm 0.7$ & $+1.7 \pm 0.7$ & $+2.8 \pm 1.2$ \\
$\Delta d_{34} / d_{0}(\%)$ & $+0.9 \pm 0.9$ & $+0.1 \pm 0.9$ & $-2.3 \pm 1.5$ \\
$\Delta d_{45} / d_{0}(\%)$ & $-0.2 \pm 0.9$ & $-0.3 \pm 0.9$ & $+0.7 \pm 1.5$ \\
$\langle u\rangle_{1}(\AA)$ & $0.16 \pm 0.02$ & $0.16 \pm 0.02$ & $0.23 \pm 0.03$ \\
$\langle u\rangle_{2}(\AA)$ & $0.13 \pm 0.02$ & $0.12 \pm 0.02$ & $0.16 \pm 0.03$ \\
$\langle u\rangle_{\text {bulk }}(\AA)$ & $0.07 \pm 0.01$ & $0.08 \pm 0.01$ & $0.09 \pm 0.02$ \\
$R_{P}$ & 0.142 & 0.130 & 0.143 \\
$\Delta E(\mathrm{eV})$ & 970 & 870 & 220 \\
$V_{0 r} \alpha(\mathrm{eV})$ & -7.24 & -7.01 & -6.51 \\
$\beta$ & 0.009 & 0.012 & $\cdots$ \\
\hline \hline
\end{tabular}


while for all reported theoretical numbers the bulk is at $T=0 \mathrm{~K}$. The reason for the deviation $(+4.3 \%$ vs $+5.8 \%$ ) from the previously reported room temperature expansion [8] lies in our improved treatment of thermal vibrations at surfaces. The mean-square displacements at the surface are determined to be a factor of 4-6 larger than in the bulk indicating that the surface is vibrationally much softer with a greatly reduced surface Debye temperature. The layer-dependent vibrational amplitudes are strongly correlated and have to be carefully optimized together with the structural parameters - this is a crucial ingredient in the LEED theory for surfaces with very different dynamical properties than the bulk. A summary of all of the optimized structural and nonstructural parameters is given in Table I. The strong enhancement of the vibrational amplitudes at the surface vs the bulk and their increase with temperature are shown in Fig. 2(b). The statistical errors in the structural parameters were estimated by calculating the variance of $R_{P}[16]$.

In order to understand the mechanism behind the observed large thermal expansion of $\mathrm{Be}(0001)$, we performed density-functional theory calculations using the local-density approximation (LDA) for the exchangecorrelation potential [17]. The $\mathrm{Be}$ atom is described by norm-conserving separable pseudopotentials [18] and the electron wave functions are expanded in a plane-wave basis with a kinetic energy cutoff of $20 \mathrm{Ry}$. The $\mathrm{Be}(0001)$ surface is modeled by a periodic slab geometry consisting of ten layers and a vacuum thickness corresponding to four such layers. The $\mathbf{k}$ space integration is done with

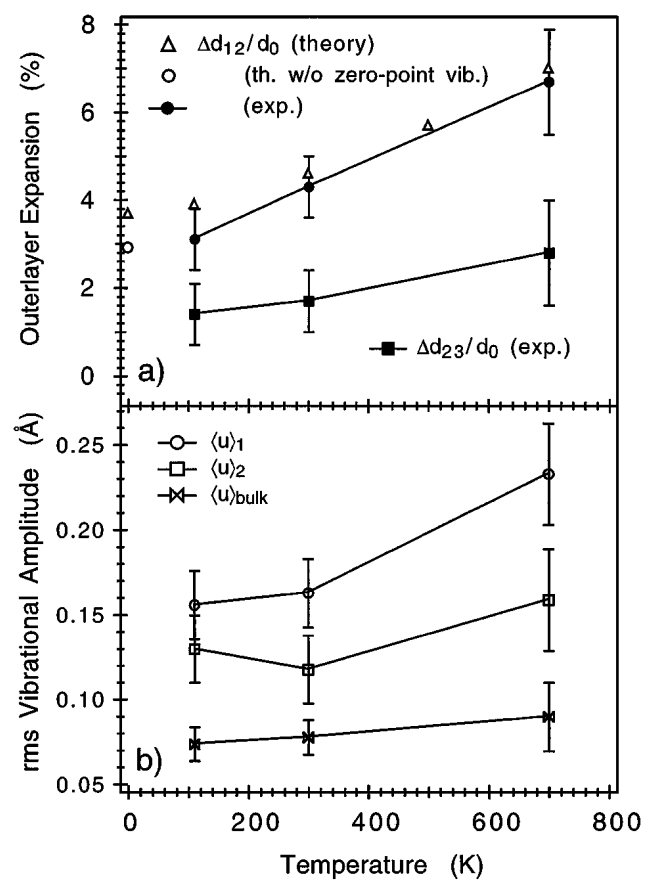

FIG. 2. (a) Change in the surface relaxation as a function of temperature from experiment (filled) and theory (open). (b) Surface vibrational amplitudes (rms motion) as a function of temperature.
30 points of the irreducible part of the surface Brillouin zone. We find that, at $T=0 \mathrm{~K}$, the first three interlayer spacings all expand, by $+2.9 \%,+1.1 \%$, and $+0.4 \%$ with respect to the bulk spacing, in good agreement with a previous pseudopotential calculation [9] and the extrapolation of our experimental results to $0 \mathrm{~K}$. The effect of zero-point vibrations is not included in these numbers.

We investigate temperature effects on the surface relaxation by considering the static and vibrational contributions to the free energy [6,7]. In the quasiharmonic approximation, the free energy for the surface is given by minimizing the function $F\left(d_{12}, T\right)$ with respect to $d_{12}$,

$$
F\left(d_{12}, T\right)=V\left(d_{12}\right)+k_{B} T \sum_{i} \ln \left\{2 \sinh \left(\frac{\hbar \omega_{i}\left(d_{12}\right)}{2 k_{B} T}\right)\right\} .
$$

The first term in Eq. (1) is the first interlayer potential and the second term is the vibrational energy and entropy; $\hbar \omega_{i}\left(d_{12}\right)$ denotes the vibrational frequencies and the summation runs over all occupied bands and $\mathbf{k}$ points. We obtain the potential energy $V$ as a function of the first interlayer spacing with the deeper spacings kept at their $T=0 \mathrm{~K}$ values. The calculated interlayer potential $V\left(d_{12}\right)$ is shown in Fig. 3. In approximation, we evaluate the sum in Eq. (1) by using three surface "wave packets" in which the top layer atoms move as a whole along the [1000], [0110], and [0001] directions on a rigid substrate. Note that these effective vibrational modes correspond to the modes at the Brillouin zone center for the top layer on a rigid substrate. The frequencies of the inplane vibrational modes can be obtained by using the frozen-phonon approach, while the frequency of the outof-plane vibrational mode is given by the curvature of $V\left(d_{12}\right)$. Considering these three surface wave packets, we find that thermal vibrations change the surface relaxation $\Delta d_{12} / d_{0}$ from $+3.7 \%$ at $0 \mathrm{~K}$ to $+3.9 \%$ at $100 \mathrm{~K},+4.6 \%$ at $300 \mathrm{~K}$, and $+7.0 \%$ at $700 \mathrm{~K}$. The theoretical value at $0 \mathrm{~K}$ is obtained by including the zero-point vibration which increases the top layer spacing from the value given by the minimum of the total energy, $+2.9 \%$, to $\Delta d_{12} / d_{0}=+3.7 \%$. For comparison, the contribution to

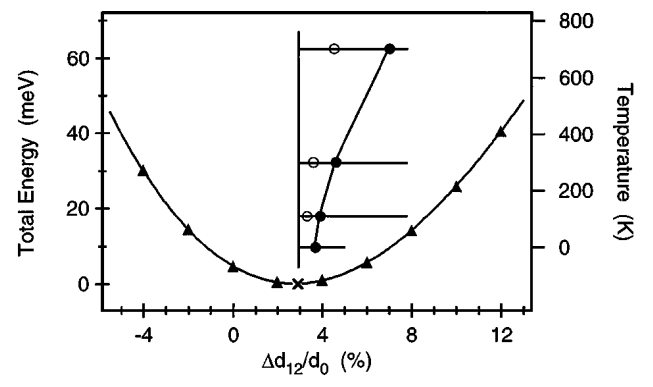

FIG. 3. Total energy per surface atom as a function of the top layer relaxation (triangles), and the top layer relaxation as a function of temperature (filled circles). The open circles represent only the contribution of the out-of-plane vibration to the thermal expansion. " $\times$ " marks the top layer relaxation at $T=0 \quad \mathrm{~K}$ neglecting zero-point vibrations. 
the thermal expansion from the above mentioned out-ofplane and in-plane vibrations is shown in Fig. 3. We find that it is largely the softening of the in-plane modes that contribute to the large thermal expansion of $\mathrm{Be}(0001)$.

Despite our rather simple model calculations, the agreement between experiment and theory for the thermal expansion of $\mathrm{Be}(0001)$ is remarkable. We note that the displacements considered in our theory do not relate to any of the actual normal modes at the surface, but rather fall in the continuum of bulklike states at $\mathbf{q}=0$. The issue of how to best incorporate the lattice vibrational modes into the calculation of thermal expansion has been addressed in recent papers: Kara et al. [19] and Xie and Scheffler [20] used selected phonon modes at the high symmetry points in the surface Brillouin zone and Lazzeri and de Gironcoli [21] used the full phonon density of states. Surely, the final answer is not in, but our experiment has shown that large thermal expansion can occur at a surface even if the interlayer potential shows very little anharmonicity. This is due to the fact that the in-plane vibrations are more important to the surface thermal expansion than the out-of-plane vibrations, as clearly shown by our calculations.

In addition to the issue of modeling the phonon density of states, there are thermal effects not represented in Eq. (1) that could be significant. As the temperature increases, the electrons above the Fermi energy extend farther out into the vacuum which can create an electrostatic force on the surface atoms, i.e., the temperature dependence in the work function. In addition, for surfaces with an abundance of surface states at the Fermi energy, two dimensional Friedel oscillations create a long range screening mechanism not present in the bulk. These surface Friedel oscillations are damped exponentially with increasing temperature due to electron-phonon scattering, leading to a temperature-dependent force constant. Balasubramanian et al. [22] recently measured an anomalously large electron-phonon coupling constant for the $\mathrm{Be}(0001)$ surface. Presumably, our LDA calculations reproduce the screening at $T=0 \mathrm{~K}$, however, they do not account for the temperature-dependent electron-phonon interaction.

In summary, we found a strong temperature dependence of the relaxation at the $\mathrm{Be}(0001)$ surface. LEED $I-V$ measurements detect a thermal expansion of the first interlayer spacing of $+3.1 \%( \pm 0.7)$ at $110 \mathrm{~K},+4.3 \%( \pm 0.7)$ in $300 \mathrm{~K}$, and $+6.7 \%( \pm 1.2)$ at $700 \mathrm{~K}$, in very good agreement with the present free energy calculation result. Thus, we found a large surface coefficient of thermal expansion of $\alpha_{s}=70 \times 10^{-6} \mathrm{~K}^{-1}, 6$ times the bulk value of $\alpha_{b}=12 \times 10^{-6} \mathrm{~K}^{-1}$ without any observable sign of anharmonicity in the interlayer potential. Moreover, our theory shows that the softening of the in-plane vibrations gives a notable contribution to the thermal expansion.

This work was partly supported by the New Energy Technology Development Organization and by NSFDMR-9510131. Part of this study was conducted at
ORNL and supported by the U.S. Department of Energy under Contract No. DE-AC05-96OR22464 with Lockheed Martin Energy Research Corp.

*Present address: Sandia National Laboratories, MS 9161, Livermore, CA 96551-0969.

†Present address: Solid State Division, Oak Ridge National Laboratory, Oak Ridge, TN 37831-6032.

[1] J. W. M. Frenken, R. G. Smeenk, and J.F. van der Veen, Surf. Sci. 135, 147 (1983); I. K. Robinson, E. Vlieg, H. Hornis, and E. H. Conrad, Phys. Rev. Lett. 67, 1890 (1991); J. W. M. Frenken and J.F. van der Veen, Phys. Rev. Lett. 54, 134 (1985).

[2] R. E. Allen and F.W. de Wette, Phys. Rev. 179, 873 (1969); S. K. S. Ma, F. W. de Wette, and G. P. Alldredge, Surf. Sci. 78, 598 (1978); C. S. Jayanthi, E. Tosatti, and L. Pietronero, Phys. Rev. B 31, 3456 (1985).

[3] J. W. M. Frenken, F. Huussen, and J.F. van der Veen, Phys. Rev. Lett. 58, 401 (1987).

[4] Y. Cao and E. Conrad, Phys. Rev. Lett. 65, 2808 (1990); P. Statiris, H.C. Lu, and T. Gustafsson, Phys. Rev. Lett. 72, 3574 (1994); G. Helgesen, D. Gibbs, A. P. Baddorf, D. M. Zehner, and S. G. J. Mochrie, Phys. Rev. B 48, 15320 (1993).

[5] A. P. Baddorf and E. W. Plummer, Phys. Rev. Lett. 66, 2770 (1991).

[6] S. Narasimhan and M. Scheffler, Z. Phys. Chem. 202, 253 (1997).

[7] J.-H. Cho and M. Scheffler, Phys. Rev. Lett. 78, 1299 (1997).

[8] H. L. Davis, J. B. Hannon, K. B. Ray, and E. W. Plummer, Phys. Rev. Lett. 68, 2632 (1992).

[9] R. Stumpf and P. J. Feibelman, Phys. Rev. B 51, 13748 (1995).

[10] N. A. W. Holzwarth and Y. Zeng, Phys. Rev. B 51, 13653 (1995).

[11] J. B. Hannon, Ph.D. thesis, University of Pennsylvania, 1994.

[12] J. B. Pendry, Low Energy Electron Diffraction (Academic, London, 1974); M. A. Van Hove and S. Y. Tong, Surface Crystallography by LEED (Springer-Verlag, Berlin, 1979).

[13] A. Barbieri and M. A. Van Hove (private communication).

[14] P. J. Rous, Prog. Surf. Sci. 39, 3 (1992); M. A. Van Hove et al., Surf. Sci. Rep. 19, 191 (1993).

[15] J. B. Pendry, J. Phys. C 13, 937 (1980).

[16] J. B. Hannon, K. Pohl, P. J. Rous, and E. W. Plummer, Surf. Sci. 364, L617 (1996).

[17] D. M. Ceperley and B. J. Alder, Phys. Rev. Lett. 45, 566 (1980); J. P. Perdew and Z. Zunger, Phys. Rev. B 23, 5048 (1981).

[18] L. Kleinman and D. M. Bylander, Phys. Rev. Lett. 48, 1425 (1982); N. Troullier and J. L. Martins, Phys. Rev. B 43, 1991 (1991).

[19] A. Kara, P. Staikov, A. N. Al-Rawi, and T.S. Rahman, Phys. Rev. B 55, R13 440 (1997).

[20] J. Xie and M. Scheffler, Phys. Rev. B 57, 4768 (1998).

[21] M. Lazzeri and S. de Gironcoli (to be published).

[22] T. Balasubramanian, E. Jensen, X.L. Wu, and S.L. Hulbert (to be published).

[23] International Tables for X-Ray Crystallography (Kynoch, Birmingham, England, 1962), Vol. III. 